\title{
Renormalized jellium model for colloidal mixtures
}

\author{
María Isabel García de Soria \\ Física Teórica, Universidad de Sevilla, Apartado de Correos 1065, E-41080, Sevilla, Spain
}

Carlos E. Álvarez

Facultad de Ciencias Naturales y Matemáticas, Universidad del Rosario, Calle 12C No. 6-25, Bogotá, Colombia

\author{
Emmanuel Trizac \\ LPTMS, CNRS, Univ. Paris-Sud, Université Paris-Saclay, 91405 Orsay, France \\ (Received 8 August 2016; published 24 October 2016)
}

\begin{abstract}
In an attempt to quantify the role of polydispersity in colloidal suspensions, we present an efficient implementation of the renormalized jellium model for a mixture of spherical charged colloids. The different species may have different size, charge, and density. Advantage is taken from the fact that the electric potential pertaining to a given species obeys a Poisson's equation that is species independent; only boundary conditions do change from one species to the next. All species are coupled through the renormalized background (jellium) density, that is determined self-consistently. The corresponding predictions are compared to the results of Monte Carlo simulations of binary mixtures, where Coulombic interactions are accounted for exactly, at the primitive model level (structureless solvent with fixed dielectric permittivity). An excellent agreement is found.
\end{abstract}

DOI: 10.1103/PhysRevE.94.042609

\section{INTRODUCTION}

Predicting structural and thermodynamic properties of charged colloidal suspensions is a difficult task [1-4]. At the simplest level of description, the solvent is treated as a continuous medium of fixed dielectric permittivity and one discards correlation effects that prevail, as a rule of thumb, for multivalent microions and sufficiently charged colloids [3]. Viewing the microionic fluid as an inhomogeneous ideal gas leads to the Poison-Boltzmann theory. However, as such, it does not easily lend itself to numerical investigations [5,6], not to mention analytical progress. In practice, this mean-field theory often needs a further mean-field-like reduction, to predict quantities that can be compared to experiments or simulations, such as osmotic pressures. One successful and popular such simplification is the so-called cell model, where an $N$-body colloidal situation is mapped onto a one-body problem, placed at the center of a Wigner-Seitz cell [7]. This cell is often taken spherical for simplicity, with a volume equal to the mean volume per colloid. As an alternative to the cell picture, a renormalized jellium model was proposed in Ref. [8], elaborating on an idea put forward by BeresfordSmith et al. [9], who nevertheless did not implement the renormalization procedure, which turns out crucial [8,10-12].

For monodisperse colloidal suspensions, both cell and jellium models yield very close and accurate results for quantities that can be compared against numerical simulations or experiments [8,13-16]. Yet, when it comes to colloidal mixtures, the cell model is not free of ambiguities [17], whereas the jellium model admits a natural extension [11,12]. In light of the intrinsic interest in polydisperse suspensions [18-21], our goal here is threefold. First, we present in Sec. II the main ingredients of the jellium model, together with a procedure that allows us to solve the problem self-consistently for mixtures, in a more efficient way than hitherto proposed. Compared to the method used in Refs $[8,10]$ for monodisperse colloids, an elegant reformulation was reported in Refs. [11,12] that significantly speeds up the resolution. We shall argue that this reformulation loses its suitability when dealing with mixtures. Second, we discuss in Sec. III some of the main features of effective charges as emerging within the jellium approach. Yet, such quantities, interesting in their own right, can be coined as "secondary," in the sense that they are often not directly measured in an experiment or in a simulation. We therefore implement Monte Carlo simulations of a binary charged mixture, which provide an important benchmark against which the polydisperse cell and the jellium schemes can be confronted. Our simulations, at the level of the primitive model, do not rely an any mean-field hypothesis, and treat exactly the Coulombic nature of the interactions between all species (colloids and microions). Conclusions are finally drawn in Sec. IV.

For the following discussion, it seems appropriate to revisit briefly an aspect of the common phenomenology of cell and jellium effective charges. For highly charged colloids [yet in the mean-field regime, where a Poisson-Boltzmann description may hold], the strong interactions between the colloids and the microions induce an accumulation of the latter in the vicinity of the colloids. This in turn induces a renormalization of the colloidal effective charge [1,3,22-24]. If the colloidal bare charge $Z_{\text {bare }}$ is large, the effective charge become independent of $Z_{\text {bare }}$; this is the saturation phenomenon [25], a signature of mean-field, where the effective charge becomes $Z_{\text {sat }}$, which only depends on the density and salt content. For a reason to become clear below, in the no salt case, $Z_{\text {sat }}$ as a function of density (or volume fraction $\eta$ ) exhibits a nonmonotonous behavior, very close to that of the function $f(X, \infty)$ versus $X$ in Fig. 1. For small $\eta$ (equivalently, small $X$ in Fig. 1), the effective charge decreases with increasing $\eta$. This is an entropy effect, whereby a lowering of $\eta$ induces a dilution of microions, which leave the vicinity of the colloids to gain translational entropy [26]. In other words, increasing $\eta$, less volume is available for the microions, electrostatic "binding" is stronger, and $Z_{\text {sat }}$ consequently decreases. However, further 


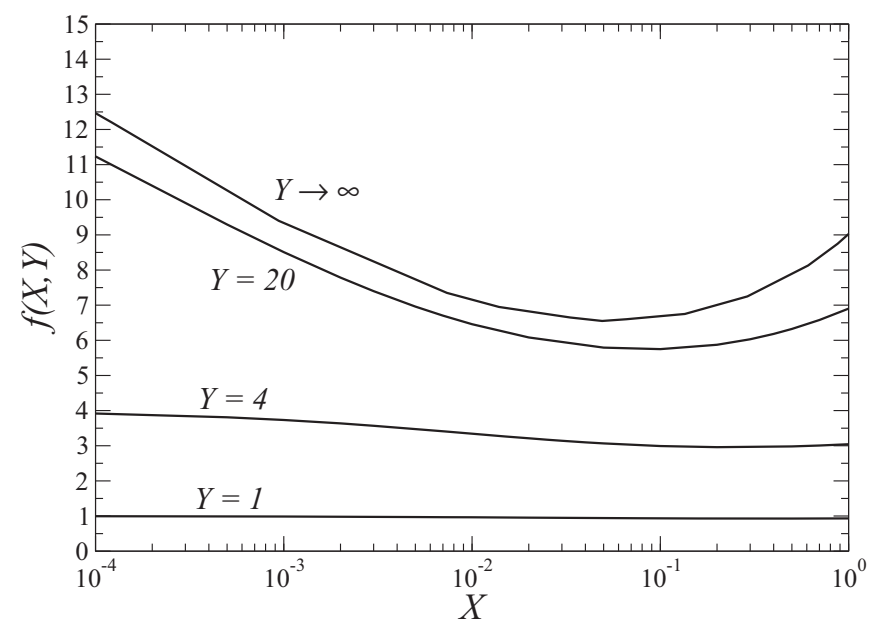

FIG. 1. Behavior of the effective charge $f(X, Y)$ as a function of screening, as encoded in $X$. The quantity $Y$ denotes the bare charge of the macroion under study, so that the upper curve, showing $f(X, \infty)$ corresponds to the saturation value studied in Ref. [23]. Practically, $f(X, Y) a / \ell_{B}$ is the effective jellium charge of a sphere having radius $a$, bare charge $Y a / \ell_{B}$, at a packing fraction $X / f(X, Y)$ (monocomponent case).

increasing $\eta, Z_{\text {sat }}$ starts to increases: this can be viewed as an indirect effect of screening. The microions efficiently screen their own interactions with the colloids, so that electrostatic binding is weakened. This dichotomy between the entropydominated and the energy-dominated regimes will be met again below, where it induces a nontrivial dependence on mixture composition.

\section{THE RENORMALIZED JELLIUM: PRINCIPLES AND RESOLUTION}

\section{A. A (mean-field $)^{2}$ approach}

We consider an arbitrary mixture of positively charged spherical colloids, where each species is indexed by an integer $i$. The radius of species $i$ having number density $\rho_{i}$ is $a_{i}$, while $e Z_{\text {bare }}^{i}$ stands for the bare charge, $e>0$ being the elementary charge. The total density is $\rho=\sum_{i} \rho_{i}$, and to characterize the composition of the mixture, it is convenient to introduce the molar fraction $x_{i}=\rho_{i} / \rho$, such that $\sum_{i} x_{i}=1$. The starting point of the jellium model is the same as the celebrated Poisson-Boltzmann theory [1,3], with an additional assumption that allows us to restrict the problem to a single-colloid formulation (the cell model approach also aims at a similar restriction but proceeds very differently [17]). The key point in the jellium approach is that the charge of other colloids around a given tagged macroion is smeared out to form a homogeneous background of charge density $\rho e Z_{\text {back }}$, in which the small ions are then immersed. A self-consistency requirement connects this background charge with the effective charge of the various species; see Refs. [8,10-12] for more details.

We denote the Bjerrum length by $\ell_{B}$, and we restrict for the sake of the argument to salt-free systems (see Sec. IIC for the general case). The dimensionless electrostatic potential around a given colloid of type $i$, centered at position $\mathbf{r}=0$, then obeys [8,10-12]

$$
\nabla^{2} \phi_{i}=4 \pi \ell_{B} \rho Z_{\mathrm{back}}\left(e^{\phi_{i}}-1\right),
$$

with boundary conditions

$$
\begin{aligned}
\phi_{i} \rightarrow 0 \text { for } r & \equiv|\mathbf{r}| \rightarrow \infty \quad \text { and } \\
\frac{d \phi_{i}}{d r} & =-Z_{\text {bare }}^{i} \ell_{B} \frac{1}{a_{i}^{2}} \text { at } r=a_{i} .
\end{aligned}
$$

The first contribution on the right-hand side of Eq. (1) stems from the counter-ions and takes the usual Poisson-Boltzmann form, while the second is that of the smeared-out background. Self-consistency demands that [8] $\rho Z_{\text {back }}=\sum_{i} \rho_{i} Z_{\text {eff }}^{i}$, where $Z_{\text {eff }}^{i}$ is the effective charge of species $i$, defined from the far-field (large $r$ ) behavior of $\phi_{i}$ [23]. Since all species obey the same differential equation, but with different boundary conditions, it follows that their effective charge is given by a unique two-parameter function $f$,

$$
\frac{Z_{\mathrm{eff}}^{i} \ell_{B}}{a_{i}}=f\left(X_{i}, \frac{Z_{\mathrm{bare}}^{i} \ell_{B}}{a_{i}}\right),
$$

where

$$
X_{i}=4 \pi \ell_{B} \rho Z_{\mathrm{back}} a_{i}^{2} / 3
$$

is a dimensionless parameter that scales like $a_{i}^{2}$ from one species to the next. The reason for including the factor 3 in the definition of $X_{i}$ will become clear below. Once the function $f(X, Y)$ is known, the self-consistency condition determines $Z_{\text {back }}$ :

$$
\begin{aligned}
Z_{\mathrm{back}} & =\sum_{i} x_{i} Z_{\mathrm{eff}}^{i} \\
& =\frac{1}{\ell_{B}} \sum_{i} x_{i} a_{i} f\left(4 \pi \ell_{B} \rho Z_{\mathrm{back}} a_{i}^{2} / 3, \frac{Z_{\mathrm{bare}}^{i} \ell_{B}}{a_{i}}\right) .
\end{aligned}
$$

At this stage, it can be appreciated that the renormalized jellium model is a mean-field simplification of an otherwise mean-field (Poisson-Boltzmann) starting point. The $N$-body Poisson-Boltzmann problem is a notoriously difficult problem to solve from a computational viewpoint (not speaking of the lack of analytical results) [6]. With the renormalized jellium, a complex mixture problem is mapped onto a series of single colloid equations, Eq. (1), in a common background with density $\rho Z_{\text {back }}$ to which all species contribute [see Eq. (5)], acting thereby as a coupling term.

\section{B. Self-consistent resolution}

In the subsequent analysis, we will single out species 1 , and use its radius $a_{1}$ as our reference length scale. Since colloidal charges appear in conjunction with the ratio $\ell_{B}$ over some radius in most expressions, we introduce the rescaled charges:

$$
\widetilde{Z}_{i}=\frac{Z_{i} \ell_{B}}{a_{1}} .
$$

Then, $X_{1}$ can be naturally expressed as a function of $\widetilde{Z}_{\text {eff }}^{1}$ and of a dressed packing fraction,

$$
\tilde{\eta}=\frac{4 \pi}{3} \rho a_{1}^{3}
$$


leading to

$$
X_{1}=\widetilde{\eta} \widetilde{Z}_{\text {back }}
$$

The dressed fraction $\tilde{\eta}$ is connected to the packing fraction $\eta$ in the suspension through

$$
\eta=\sum_{i} \frac{4 \pi}{3} \rho_{i} a_{i}^{3}=\tilde{\eta} \sum_{i} x_{i} \frac{a_{i}^{3}}{a_{1}^{3}} .
$$

To summarize the previous discussion, the key equation to be solved within the jellium model is

$\widetilde{Z}_{\text {back }} \equiv \frac{Z_{\text {back }} \ell_{B}}{a_{1}}=\sum_{i} x_{i} \frac{a_{i}}{a_{1}} f\left(\widetilde{\eta} \widetilde{Z}_{\text {back }} \frac{a_{i}^{2}}{a_{1}^{2}}, \widetilde{Z}_{\text {bare }}^{i} \frac{a_{1}}{a_{i}}\right)$.

Hence, once the physical parameters have been chosen (bare charges, compositions $x_{i}$, radii $a_{i}$, and packing fraction), one needs to find the root $X^{*}$ of equation

$$
\frac{X}{\tilde{\eta}}=\sum_{i} x_{i} \frac{a_{i}}{a_{1}} f\left(X \frac{a_{i}^{2}}{a_{1}^{2}}, \widetilde{Z}_{\text {bare }}^{i} \frac{a_{1}}{a_{i}}\right),
$$

from which the background (effective) charge follows: $\widetilde{Z}_{\text {back }}=$ $X^{*} / \tilde{\eta}$. Of course, the function $f\left(x, \widetilde{Z}_{\text {bare }}^{i} a_{1} / a_{i}\right)$ should be computed beforehand for all species, but this task deals with a monocomponent problem only. In other words, $f(X, Y)$ is the effective charge of the potential $\phi$ obeying

$$
\frac{d^{2} \phi}{d \tilde{r}^{2}}+\frac{2}{\tilde{r}} \frac{d \phi}{d \tilde{r}}=3 X\left(e^{\phi}-1\right)
$$

with boundary conditions

$$
\phi \rightarrow 0 \text { for } \tilde{r} \rightarrow \infty \quad \text { and } \quad \frac{d \phi}{d \tilde{r}}=-Y \text { at } \tilde{r}=1,
$$

meaning that for large $\tilde{r}$,

$$
\phi \sim f(X, Y) \frac{e^{-\tilde{\kappa}(\tilde{r}-1)}}{(1+\tilde{\kappa}) \tilde{r}} \quad \text { with } \quad \tilde{\kappa}^{2}=3 X
$$

It is thus straightforward to obtain $f$, following, for instance, the method presented in the Appendix of Refs. [24,27]. Typical results are shown in Fig. 1. When $Y$ is small, charge renormalization effects disappear, so that $f(X, Y)=Y$, irrespective of $X$. In the limit of small bare charges, the background charge thus takes a simple form: $Z_{\text {back }}=\sum_{i} x_{i} Z_{\text {bare }}^{i}$. On the other hand, upon increasing the bare charge through $Y$, the effective charge also increases, with always $f<Y$ [28]. The saturation upper curve is reached for large $Y$.

It appears at this point that the packing fraction (either the real one, $\eta$, or its dressed counterpart $\widetilde{\eta}$ ) only enters the self-consistency condition on the left-hand side of Eq. (11). As a consequence, our method allows us to treat very simply the effect of packing fraction, since the more time-consuming part of the calculation is that of the right-hand side of Eq. (11). This is an important advantage over previous proposals, be it the technique presented in Ref. [10] or subsequent improvements $[11,12]$.

For concreteness, the explicit solution of a binary colloidal problem is constructed in Fig. 2 with relatively weakly charged macroions: both have the same charge $\widetilde{Z}_{\text {bare }}^{1}=\widetilde{Z}_{\text {bare }}^{2}=4$, but they differ in size: $a_{2} / a_{1}=2$. The pristine effective charges $f(X, 4)$ and $f(X, 2)$ should be known, from which one

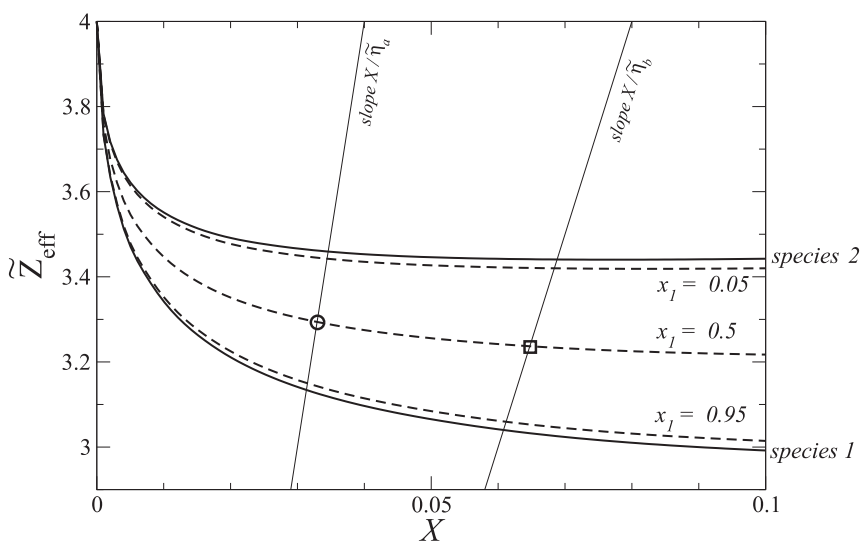

FIG. 2. Illustration of the method employed to find the solution of Eq. (11), for $\widetilde{Z}_{\text {bare }}^{1}=\widetilde{Z}_{\text {bare }}^{2}=4, a_{2}=2 a_{1}$, and $\tilde{\eta}=10^{-2}$. The continuous curves show the effective charge $f(X, 4)$ (lower curve, indexed "species 1 ") and $2 f(4 X, 2)$ (upper curve, indexed "species 2 "). Depending on the mixture composition, the weighted average of both with weights $x_{1}$ and $x_{2}=1-x_{1}$ are shown with the dashed lines. These are the master curves, corresponding to the right-hand side of Eq. (11), to be considered for all possible $\widetilde{\eta}$. The linear curves show $X / \widetilde{\eta}$ for two values of the dressed packing fraction $\left(\tilde{\eta}_{a}=10^{-2}\right.$ and $\left.\tilde{\eta}_{b}=2 \times 10^{-2}\right)$. For an equimolar mixture $\left(x_{1}=1 / 2\right)$, the effective background charge is shown, by the circle (case $\tilde{\eta}=10^{-2}$ ) and by the square (case $\widetilde{\eta}=2 \times 10^{-2}$ ).

constructs the weighted average appearing in the right-hand side of Eq. (10) is calculated. Depending on the mixture composition, this leads to the dashed curves: from bottom to top are a species 1-rich, an equimolar, and a species 2-rich mixture. The procedure closes, after the choice of density through $\tilde{\eta}$, by searching for the intersection with the line $\underset{\widetilde{Z}}{X} \tilde{\eta}$. With $x_{1}=1 / 2$, we thereby get the background charge $\widetilde{Z}_{\text {eff }}=3.29$ at $\tilde{\eta}=10^{-2}$, and $\widetilde{Z}_{\text {eff }}=3.23$ at $\widetilde{\eta}=2 \times 10^{-2}$. The graphical construct proposed allows us to anticipate the dependence of effective charges on mixture composition; see Fig. 3, which corresponds to a bidisperse solution with $a_{1}=a_{2}$ but unequal bare charges. It can be expected that increasing $\eta$, a regime will be reached in the vicinity of the species 2 curve minimum, where the corresponding range for the variations of $Z_{\text {sat }}^{2}$ with composition will vanish. This will be confirmed in Sec. III. Turning to the effect of binary mixture composition on background charge in the case of unequal colloidal sizes, Figs. 4 and 5 address large bare charges (saturated limit) and show by vertical dashed lines how $X$ is affected by going from $x_{1}=1$ to $x_{1}=0$. Once $X$ (or more precisely, the root $X^{*}$ ) is known, the background charge follows from $\widetilde{Z}_{\text {back }}=X / \widetilde{\eta}$. These two figures are for $a_{2} / a_{1}=1 / 3$ and 3 . Of course, the $1 \leftrightarrow 2$ labeling of species is immaterial in the case $x_{1}=x_{2}=1 / 2$, so that at a given density $\rho$, the solutions of the two problems should coincide. This is not the case in Figs. 4 and 5 , since $\tilde{\eta}$ is common to both, meaning that they correspond to different densities $\rho$.

\section{The general case}

So far, the discussion focused on the deionized limit. In case salt is present, for instance when the system is in osmotic 


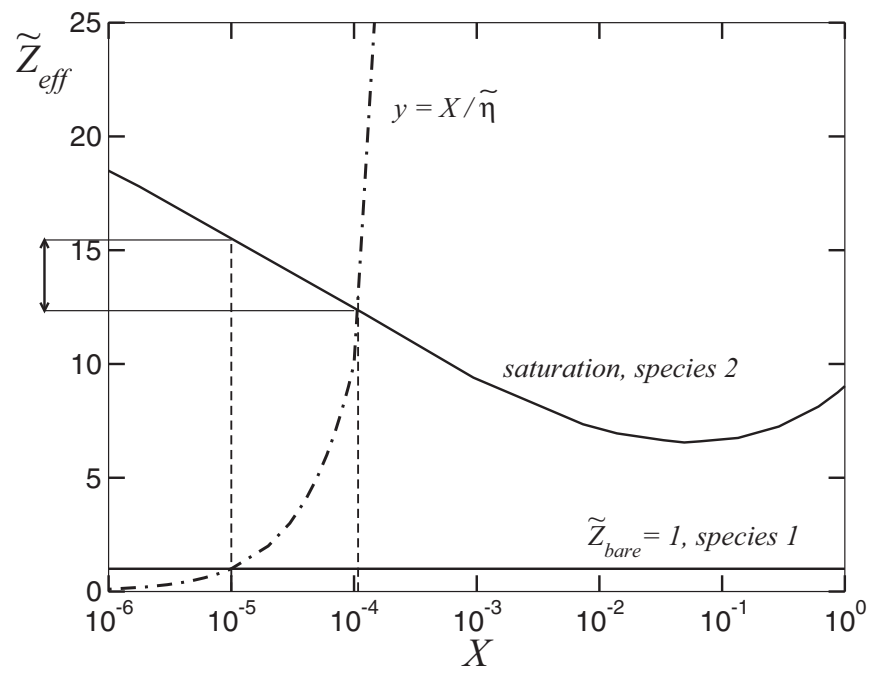

FIG. 3. Like-size binary mixture of a weakly charged species with $\widetilde{Z}_{\text {bare }}^{1}=1$ and a strongly charged species (limit $\widetilde{Z}_{\text {bare }}^{2} \rightarrow \infty$ ). The packing fraction is $\eta=\widetilde{\eta}=10^{-5}$. When changing the mixture composition, the allowed range for $\widetilde{Z}_{\text {eff }}^{2}$ is displayed by the vertical double arrow on the left-hand side).

equilibrium with a salt reservoir of density $c_{s}$, Eq. (1) becomes

$$
\nabla^{2} \phi_{i}=4 \pi \ell_{B}\left[2 c_{s} \sinh \phi_{i}-\rho Z_{\text {back }}\right],
$$

with the boundary conditions

$$
\begin{aligned}
2 c_{s} \sinh \phi_{i}-\rho Z_{\mathrm{back}} & \rightarrow 0 \text { for } r \rightarrow \infty \text { and } \\
\frac{d \phi_{i}}{d r} & =-Z_{\text {bare }}^{i} \ell_{B} \frac{1}{a_{i}^{2}} \text { at } r=a_{i} .
\end{aligned}
$$

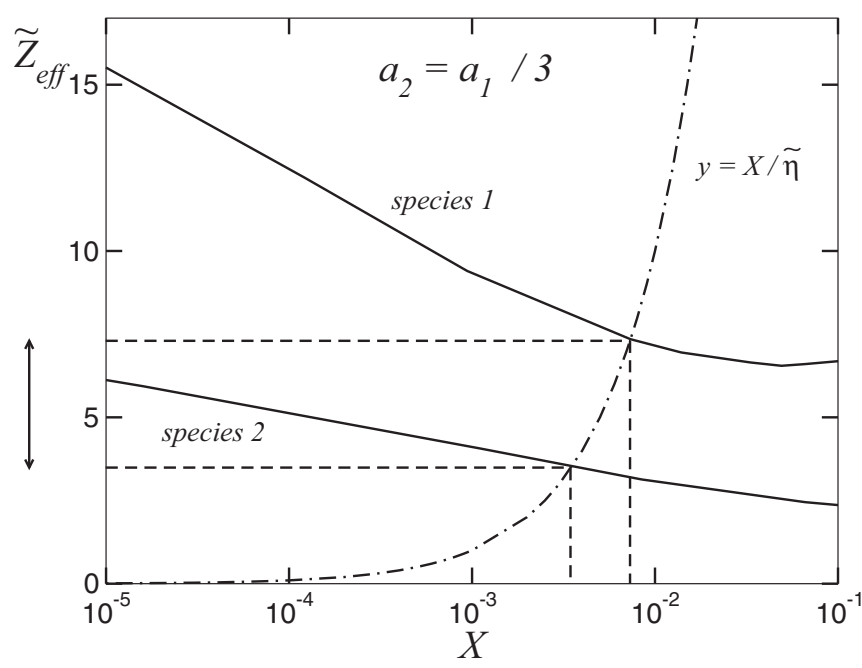

FIG. 4. Binary case. Log-linear plot. Here $\widetilde{Z}_{\text {bare }}^{1}=\widetilde{Z}_{\text {bare }}^{2}$ are both saturated (divergent), $\widetilde{\eta}=10^{-3}, a_{2} / a_{1}=1 / 3$. The weighted average Eq. (10) lies in between the two thick curves upon changing the composition $x_{1}$ from 0 (in which case it corresponds to the "species 2 " bottom curve) to 1 (in which case it coincides to the "species 1 " upper curve). As a consequence, the values $X$ can lie between the two vertical dashed lines, from which the allowed range for $\widetilde{Z}_{\text {back }}$ can be read on the $y$ axis and falls in between the two horizontal dashed lines. As in Fig. 3, the allowed range is thus shown by the vertical arrow.

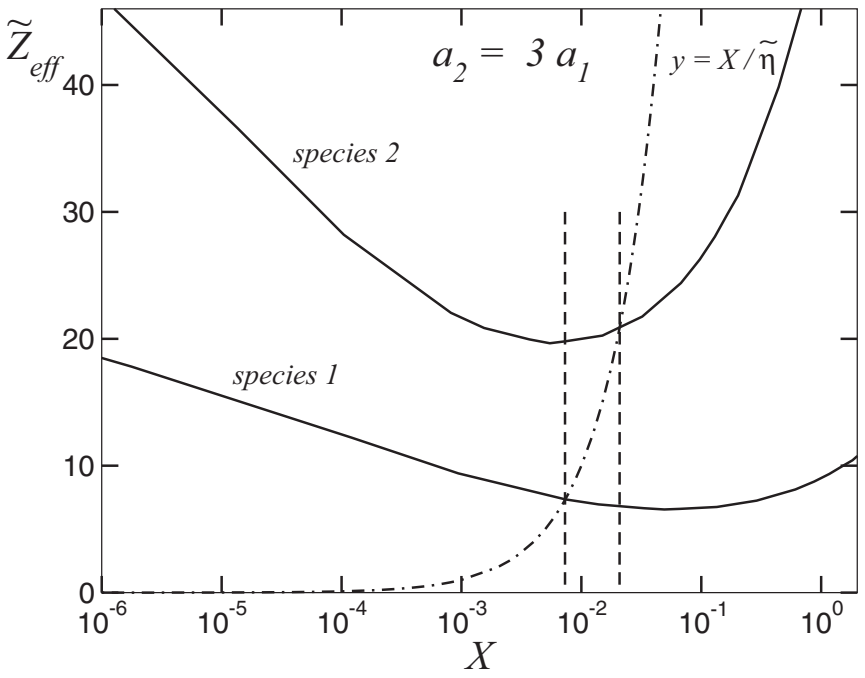

FIG. 5. Same as described in the caption of Fig. 4 but for $a_{2} / a_{1}=3$

The first equation stems from electroneutrality and defines the potential at infinity, often referred to as the Donnan potential. The second results from Gauss' theorem. Defining the inverse squared Debye length in the reservoir as $\kappa_{\text {res }}^{2}=8 \pi \ell_{B} c_{s}$, we arrive at

$$
\nabla^{2} \phi_{i}=\kappa_{\mathrm{res}}^{2} \sinh \phi_{i}-4 \pi \ell_{B} \rho Z_{\mathrm{back}},
$$

and we can proceed along very similar lines as in Sec. II A. We have assumed here the salt to be monovalent, for simplicity. Generalization to mixed-valency salts is straightforward. Expressing the colloids' effective charges requires the introduction of a generalization of function $f$, which we denote $f_{\text {salt }}$, so that

$$
\frac{Z_{\mathrm{eff}}^{i} \ell_{B}}{a_{i}}=f_{\text {salt }}\left(X_{i}, \frac{Z_{\text {bare }}^{i} \ell_{B}}{a_{i}}, \kappa_{\text {res }} a_{i}\right),
$$

keeping the same notation for $X_{i}$. Of course, one has $f(X, Y)=$ $f_{\text {salt }}(X, Y, 0)$. The self-consistency condition becomes

$$
\begin{aligned}
Z_{\mathrm{back}} & =\sum_{i} x_{i} Z_{\mathrm{eff}}^{i} \\
& =\frac{1}{\ell_{B}} \sum_{i} x_{i} a_{i} f_{\mathrm{salt}}\left(4 \pi \ell_{B} \rho Z_{\mathrm{back}} a_{i}^{2} / 3, \frac{Z_{\mathrm{bare}}^{i} \ell_{B}}{a_{i}}, \kappa_{\mathrm{res}} a_{i}\right) .
\end{aligned}
$$

Again, the functions $f_{\text {salt }}$, which are those of a single component problem, can be computed as such [24], and subsequently used to describe an arbitrary mixture. Typical results are shown in Fig. 6, for a colloidal bare charge that is neither small nor large, meaning that it is of order $10 a / \ell_{B}$.

From the very form of Eq. (17), it appears that the longdistance potential $\phi_{i}$ is of the standard form,

$$
\phi_{i}-\phi_{i}(\infty) \sim \frac{Z_{\mathrm{eff}}^{i} \ell_{B}}{\left(1+\kappa a_{i}\right)} \frac{e^{-\kappa\left(r-a_{i}\right)}}{r},
$$

an expression which can be viewed as defining the effective charge $Z_{\text {eff }}^{i}$, and which involves the effective screening length 


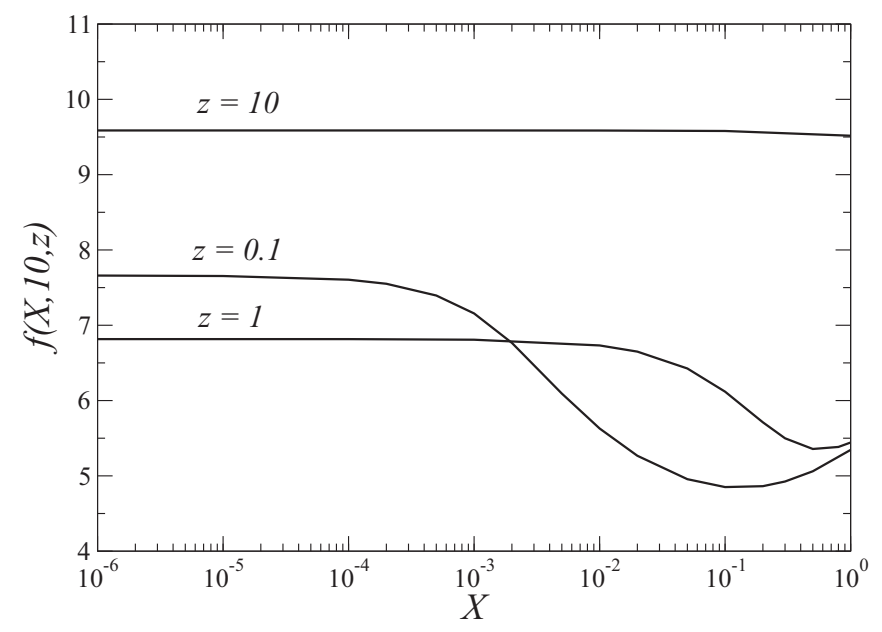

FIG. 6. Effect of salt on the screening function $f_{\text {salt }}$ appearing in Eq. (18). Here, the reduced charge in chosen equal to 10 , and we show $f_{\text {salt }}(X, 10, z)$ as a function of $X$ (the jellium background dimensionless charge), for different salinities $z$.

$\kappa^{-1}$ given by

$$
\kappa^{2}=\kappa_{\mathrm{res}}^{2} \cosh \left[\phi_{i}(\infty)\right] .
$$

This quantity can be reexpressed as

$$
\kappa^{4}=\kappa_{\text {res }}^{4}+\left(4 \pi \ell_{B} \rho Z_{\text {back }}\right)^{2} .
$$

It is worth emphasizing here that a bona fide feature of jellium-like models is that the osmotic pressure takes a particularly simple form, and is directly connected to the effective charges $[8,10,12]$ :

$$
\begin{aligned}
\beta P & =\rho+2 c_{s} \cosh \left[\phi_{i}(\infty)\right]-2 c_{s} \\
& =\rho+\sqrt{\left(2 c_{s}\right)^{2}+\left(\rho Z_{\mathrm{back}}\right)^{2}}-2 c_{s} .
\end{aligned}
$$

It is the excess pressure with respect to the salt reservoir, including the colloidal contribution, taken ideal for simplicity. For salt-free systems, it takes the form $\beta P=\rho+Z_{\mathrm{back}} \rho$, which is usually close to $Z_{\text {back }} \rho$.

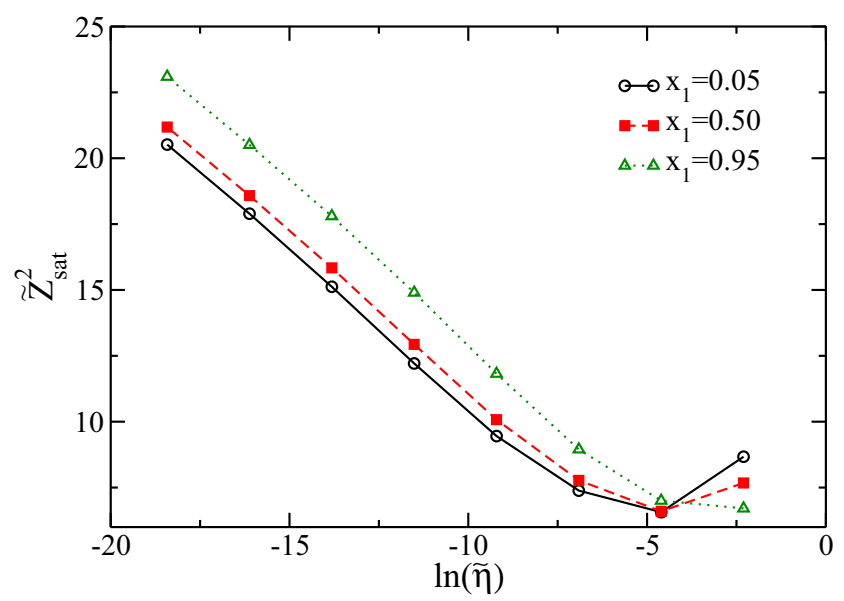

\section{Comparison with previous approaches}

Before discussing the physical results, it seems opportune to put the method described above in the context of those used so far. For the sake of the discussion, we assume that the salt content is fixed, and we wish to identify the number of independent parameters that have to be (essentially continuously) varied before the full solution is reached. This allows for a definition of the "dimensionality" of the method, a measure of user-friendliness.

We start by the monocomponent case and consider that the goal is to obtain a curve $Z_{\text {eff }}$ as a function of $Z_{\text {bare, }}$, parameterized by $\eta$. The original method used in Refs. $[8,10]$ is brute force: for each $\eta, Z_{\text {bare }}$, and $Z_{\text {back, Eq. (17) is }}$ solved by a shooting method, to obtain the desired value of $Z_{\text {bare }}$ : this is a procedure of dimension 1 [29]. Then $Z_{\text {back }}$ should be changed, to find in which case the background and effective charges coincide. In that respect, the resolution is of dimension 2 for each $\eta$ and $Z_{\text {bare, }}$, it is thus of dimension 4 overall. Castañeda-Priego and collaborators [11,12] have found an interesting reformulation, in which self-consistency is automatically enforced by imposing a priori $Z_{\text {back }}=Z_{\text {eff }}$, and computing the corresponding $Z_{\text {bare }}$ in one step only. This is achieved by constraining the far-field. For each $\eta$, the method is of dimension 1 ( $Z_{\text {eff }}$ has to be changed). Hence, the overall dimension is two, which is an improvement. Finally, with the method presented here, a unique function $f$ of two parameters encodes the relevant information, and the approach also is of dimension 2 .

The "degeneracy" between the latter two procedures is lifted when considering mixtures. Following Refs. [11,12], the effective charges have to be chosen a priori, and the bare charges follow. However, a physical problem is in practice formulated in terms of bare charges. This subtlety is immaterial for monocomponent systems: the functions $Z_{\text {eff }}\left(Z_{\text {bare }}\right)$ and $Z_{\text {bare }}\left(Z_{\text {eff }}\right)$ convey the same information and are simply connected. This is no longer the case for mixtures, where the functions $Z_{\text {eff }}^{i}\left(Z_{\text {bare }}^{1}, Z_{\text {bare }}^{2} \ldots\right)$ and $Z_{\text {bare }}^{i}\left(Z_{\text {eff }}^{1}, Z_{\text {eff }}^{2} \ldots\right)$ are not simply related. Deriving the second from the first requires a shooting task that appears quite impractical. Additionally, there is no guarantee that the a priori choices of effective charges are not unphysical, with for instance values above the

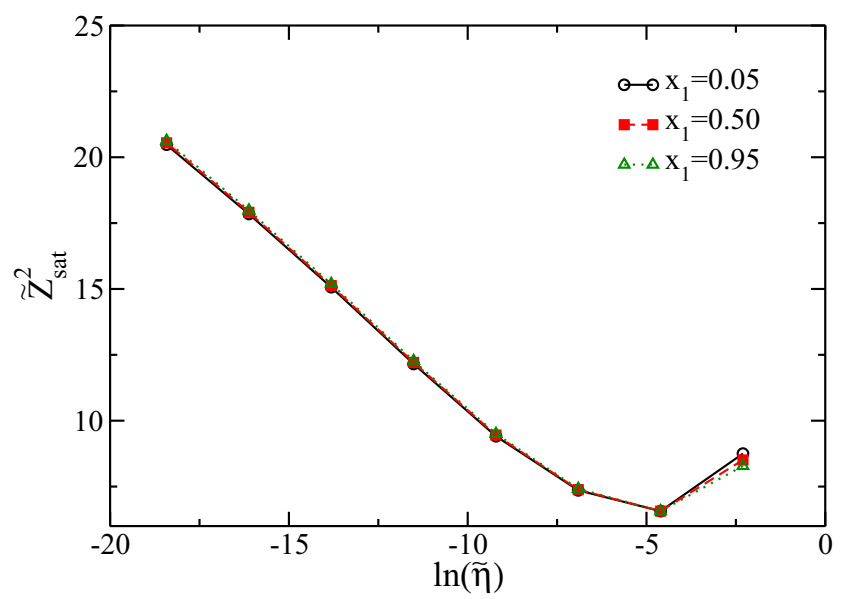

FIG. 7. Saturation charge, $\widetilde{Z}_{\text {sat }}^{2}$, as a function of the total density of colloids in the no salt case. The dependence on $x_{1}$ is not very strong. In these case $\widetilde{Z}_{\text {bare }}^{1}=1$ (left) and $\widetilde{Z}_{\text {bare }}^{1}=20$ (right). 


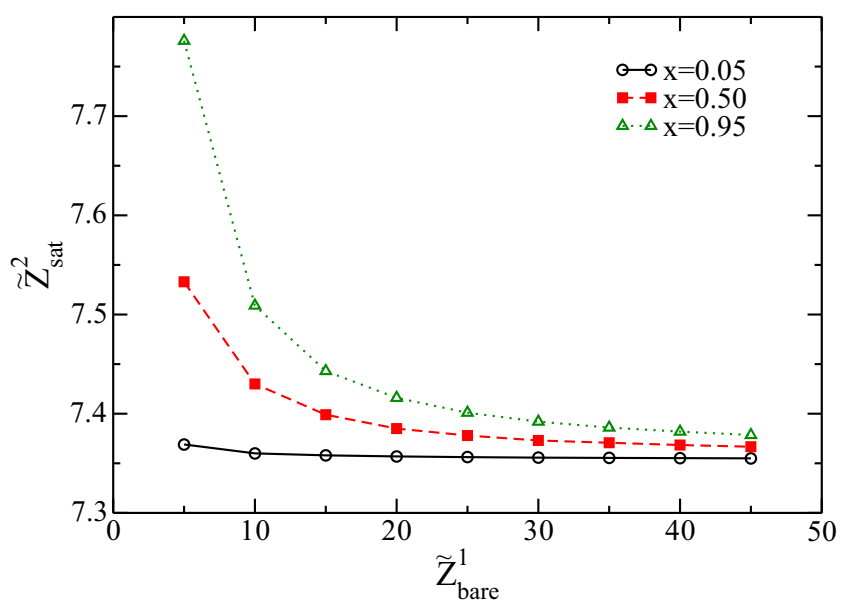

FIG. 8. Saturation charge in the no salt case as a function of $\widetilde{Z}_{\text {bare }}^{1}$ for a value of the total fraction $\tilde{\eta}=10^{-3}$ and with $a_{1}=a_{2}$ (so that $\eta=10^{-3}$ as well).

saturation limit. This is the case, for instance, in Fig. 5 of Ref. [12], for low salt content [30]. Our alternative treatment is free of these shortcomings.

\section{RESULTS}

\section{A. General features of effective charges}

In this section we focus on the behavior of the saturation charge. In Ref. [8], it has been found that the saturation value for the charge when the concentration was small $\left(\tilde{\eta}<10^{-5}\right)$ was given by

$$
Z_{\mathrm{sat}} \simeq \frac{a}{\ell_{B}}[\delta-\gamma \log (\widetilde{\eta})],
$$

where $\gamma \simeq 1$ and $\delta \simeq 2$. In Fig. 7 the saturation value $\widetilde{Z}_{\text {sat }}^{2}$ has been plotted as a function of the density $\tilde{\eta}$ for the no salt case, for $\widetilde{Z}_{\text {bare }}^{1}=1$ (left) and $\widetilde{Z}_{\text {bare }}^{1}=20$ and three values of the composition $x_{1}$. As we can see, for small values of $\tilde{\eta}$, Eq. (24) holds, with different values for $\delta$ and $\gamma$, that depends slightly on $x_{1}$. In Fig. 7 (right) we reobtain the monodisperse case because both species are of the same size and the bare charges are large enough to be in the saturation limit.

In Fig. 8, the saturation value has been plotted as a function of $\widetilde{Z}_{\text {bare }}^{1}$ for a density $\tilde{\eta}=10^{-3}$ and three values of the concentration $x_{1}$. The dependence on $x_{1}$ decreases as the value of $\widetilde{Z}_{\text {bare }}^{1}$ increases because we approach the saturation for species 1 . We are now in a position to analyze the dependence of this property on the colloidal sizes asymmetry. To this aim, we have studied the variation of the saturation value of the charge as we vary the size ratio. In Fig. 9, left, we have plotted $\widetilde{Z}_{\text {sat }}^{2}$ as a function of $a_{2} / a_{1}$ for a system with $\tilde{\eta}=10^{-3}$ and $x_{1}=0.5$. It appears that the dependence is roughly linear on $a_{2} / a_{1}$. The dashed line is a linear fitting. However, on closer inspection, the situation is more complex; see Fig. 9, right, plotting $\widetilde{Z}_{\text {sat }}^{2} a_{1} / a_{2}$ for different values of $\widetilde{Z}_{\text {bare }}^{1}$ and $x_{1}$. It can be seen that for $a_{2} / a_{1}<1$, the behavior of $Z_{\text {sat }}^{2}$ is not linear in $a_{2} / a_{1}$. This behavior can be understood from the plot of $f(X, \infty)$ reported in Fig. 1, which exhibits in its left-most part (say for $X<10^{-2}$ ), the entropy dominated regime alluded to in the introduction (decrease of the effective charge with an increase of concentration). Upon decreasing $a_{2}$ at fixed $a_{1}$, the relevant background parameter $X_{2}$ decreases as $a_{2}^{2}$, and this leads, from Eq. (3), to an increase of $Z_{\text {sat }}^{2} \ell_{B} / a_{2}$. On the other hand, increasing $a_{2}$, one probes at some point the shallow minimum seen in Fig. 1, where $f$ takes values around 7. This is compatible with Fig. 9, right, and also means that $\widetilde{Z}_{\text {sat }}^{2}=Z_{\text {sat }}^{2} \ell_{B} / a_{1}$ scales like $a_{2} / a_{1}$ (see Fig. 9, left).

\section{B. Osmotic pressure and comparison to Monte Carlo simulations}

One of the advantages of the jellium model is that once the renormalized charges are known, the evaluation of the osmotic pressure is straightforward. However, a competing theory of equal simplicity does exist [17], where the standard Poisson-Boltzmann cell model [7,22] has been generalized for mixtures. For colloidal spheres, the radii of the cells can be different for each type of macroion. These radii are determined self-consistently for a given set of parameter, from the solution of the nonlinear Poisson-Boltzmann equation with appropriate boundary conditions [17].
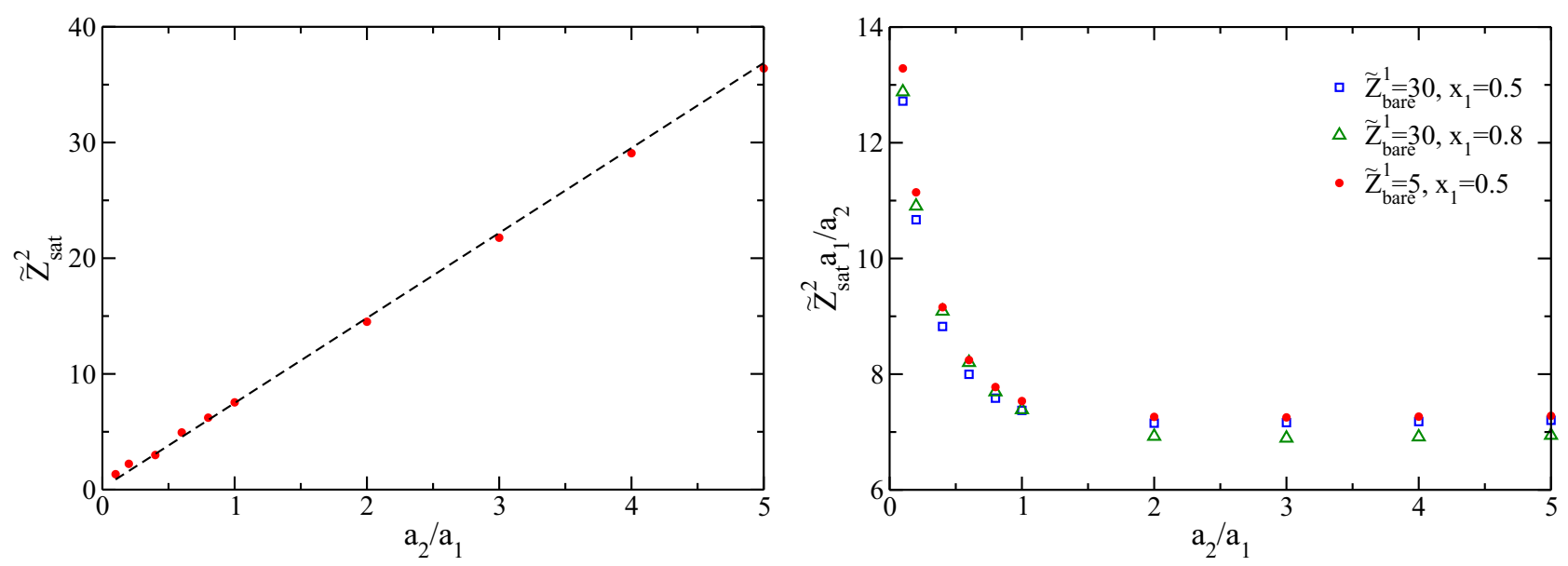

FIG. 9. (Left) Saturation charge $\widetilde{Z}_{\text {sat }}^{2}$ as a function of the radius ratio $a_{2} / a_{1}$, for a system with $\tilde{\eta}=10^{-3}, \widetilde{Z}_{\text {bare }}^{1}=5$ and $x_{1}=0.5$. (Right) $\widetilde{Z}_{\text {sat }}^{2} a_{1} / a_{2}$ for a system with $\widetilde{\eta}=10^{-3}$ and different values of $\widetilde{Z}_{\text {bare }}^{1}$ and $x_{1}$. 


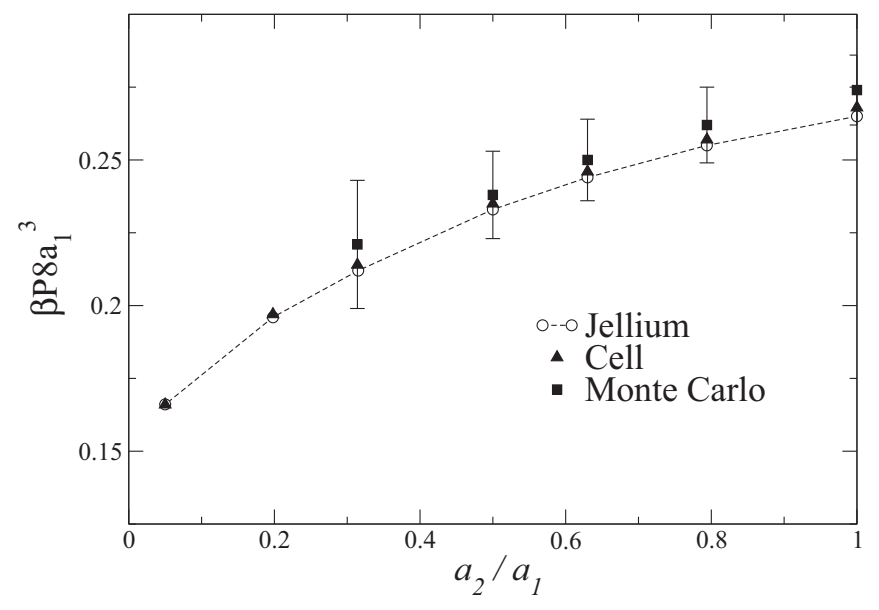

FIG. 10. Osmotic pressure for a system consisting of two kinds of colloidal particles with the same charge $\widetilde{Z}_{\text {bare }}^{i}=6.4$, as a function of $a_{2} / a_{1}$.

In this section, we compare the results from both methods, with those of Monte Carlo (MC) simulations of bidisperse systems of spherical charged colloids. Explicit counter-ions are considered, without added salt. The simulations, which treat exactly Coulombic forces, have been performed in the NVT ensemble with periodic boundary conditions. In order to take into account the long range electrostatic interactions with the images of the system, Ewald summations were used [31,32]. The number of colloidal particles of each type is $N_{1}=N_{2}=$ 40 , confined in a simulation box of side length $L$. The number of monovalent counterions, $N_{\text {ion }}$, was set in each case so that charge neutrality was obtained.

The pressure of the system was computed using the virial theorem,

$$
\beta P=\rho+\beta\langle W\rangle,
$$

where $\rho$ is the particle number density, $\beta=\left(k_{B} T\right)^{-1}$ and $W$ is the virial function,

$$
W=-\frac{1}{3 V} \sum_{i=1}^{N} \boldsymbol{r}_{i} \cdot \nabla_{i} U
$$

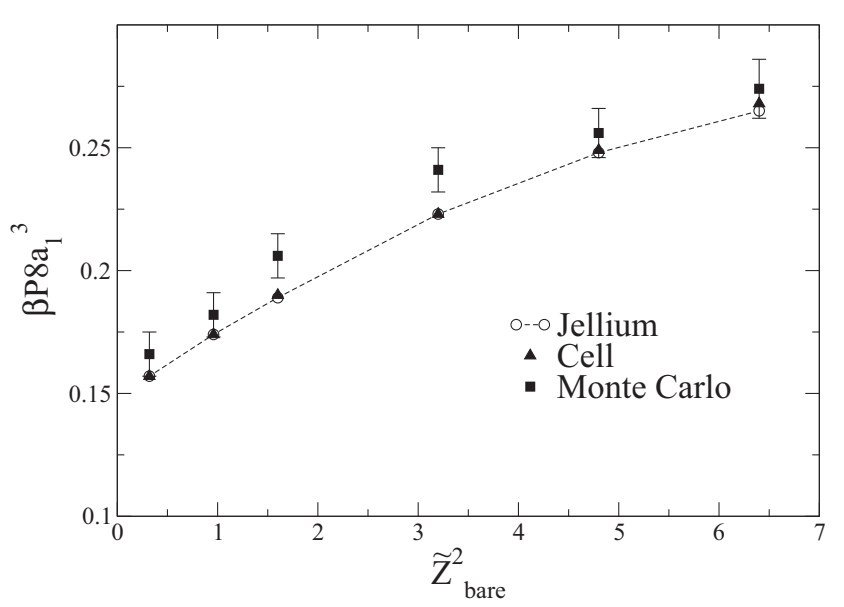

for a system with particles at positions $\boldsymbol{r}_{i}$ interacting between themselves with a pair potential $U$, which is the sum of the long-range Coulomb potential, using the known Ewald expressions [33-35] with the minimum image convention, and a short-range hard-core potential.

In order to compute $\langle W\rangle$ for the hard-core part of the potential we use [36]

$$
\beta\langle W\rangle=\frac{1}{3 V}\left\langle\sum_{i=1}^{N-1} \sum_{j>i}^{N} 2 F\left(\boldsymbol{r}_{i j}\right) \delta\left(F\left(\boldsymbol{r}_{i j}\right)-1\right)\right\rangle,
$$

where $F\left(\boldsymbol{r}_{i j}\right)$ is an overlap function. In the case of spherical particles the overlap function has a simple form and the virial expression for the hard core interaction is

$$
\beta\langle W\rangle=\frac{1}{3 V}\left\langle\sum_{i=1}^{N-1} \sum_{j>i}^{N} \frac{r_{i j}^{2}}{\sigma_{i j}} \delta\left(r_{i j}-\sigma_{i j}\right)\right\rangle,
$$

in which $\sigma_{i j}=\left(\sigma_{i}+\sigma_{j}\right) / 2$ and $\sigma_{i}$ is the diameter of particle $i$.

In all the simulations, the radius of the first colloidal species $\left(a_{1}\right)$ was kept constant and used to normalize the distances. The radius of the ions was set to $a_{\text {micro }}=10^{-3} a_{1}$. The volume of the simulation box and the Bjerrum length were also kept constant at $\left(L / a_{1}\right)^{3}=33540.8$ and $\ell_{B} / a_{1}=0.32$, respectively. The systems were equilibrated for $3 \times 10^{5} \mathrm{MC}$ steps before averaging and then the averages were carried out for $3 \times 10^{5} \sim 8 \times 10^{5}$ MC steps, where a MC step involves a test move of every particle in the system.

Three sets of simulations were carried out at $\tilde{\eta}=0.01$. In the first set, the two colloidal species have the same bare charge $\widetilde{Z}_{\text {bare }}^{1}=\widetilde{Z}_{\text {bare }}^{2}=6.4$ (and thus $Z_{\text {bare }}^{1}=20$ ), while the radius of the second species $\left(a_{2}\right)$ is varied. We show in Fig. 10, the simulation results (filled squares) as well as the predictions obtained by the renornalized jellium model (empty circles) and the cell model (filled triangles). As can be seen, the agreement between the three sets is very good. In the second set of simulations, the colloids are all of the same size $\left(a_{1}=a_{2}\right)$, the charge of the first species is kept at $\widetilde{Z}_{\text {bare }}^{1}=6.4$ and the charge of the second species $\left(\widetilde{Z}_{\text {bare }}^{2}\right)$ is varied (Fig. 11, left).

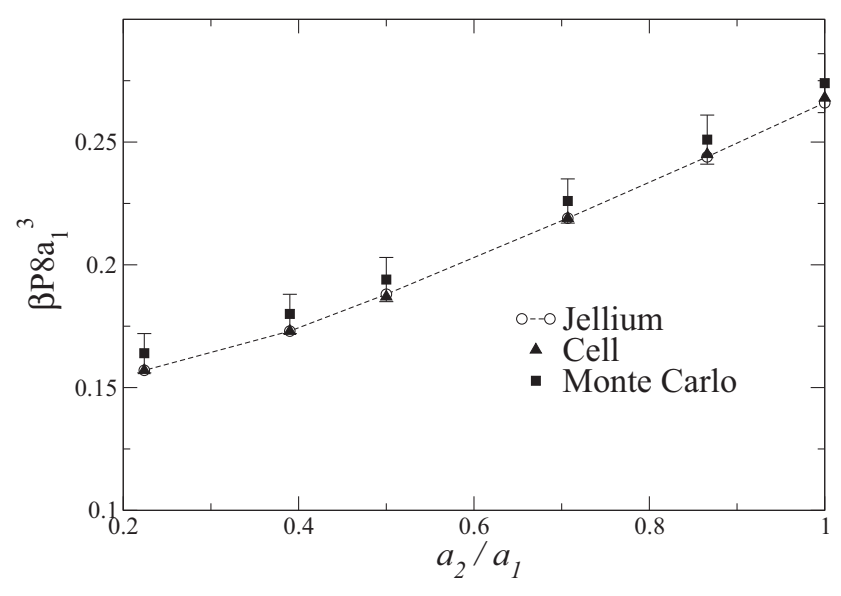

FIG. 11. (Left) Osmotic pressure for a system consisting of two kinds of colloidal particles with the same radius $a_{1}=a_{2}$, as a function of $\widetilde{Z}_{\text {bare }}^{2}$, with $\widetilde{Z}_{\text {bare }}^{1}=6.4$. (Right) Osmotic pressure, changing the size ratio, keeping a constant surface charge density for both colloids. Here, $a_{1}$ is fixed, $\widetilde{Z}_{\text {bare }}^{i}=6.4$, and $a_{2}$ changes. 
The results obtained from the jellium and cell models are again nearly identical. Although the pressure they predict is in general smaller than that of the MC simulations, the agreement is good. The situation is similar for the third set of simulations, (Fig. 11, right) in which $a_{1}$ is fixed and $a_{2}$ varies in such a way as to keep the surface charge density $\left(s_{i}\right)$ constant $s_{i}=Z_{\text {bare }}^{i} /\left(4 \pi a_{i}^{2}\right)$. In all cases, the proximity of cell and jellium results is striking, and somewhat surprising given they rely on rather distinct calculations.

The MC data shown here do not allow to discriminate one approach against the other. The reason may be that charge renormalization effects are not overwhelming with the parameters of the simulations, even if not negligible. It would be in this respect interesting to increase somewhat the values of the bare charges, to enhance nonlinear effects. In doing so though, one has to keep in mind that correlation effects will be increased as well, and when the so-called plasma parameter $\Xi_{i}=2 \pi \ell_{B}^{2} s_{i}$ exceeds unity, the whole PoissonBoltzmann-like description will start to break down, be it in its jellium, or in its cell clothing $[3,37,38]$. With the parameters of Fig. 11, right, we have $\Xi_{1}=\Xi_{2}=1$. On the other hand, with the procedure underlying Fig. 10 , we have $\Xi_{2} \simeq a_{1} / a_{2}$ and, therefore, decreasing $a_{2} / a_{1}$, Coulombic correlations increase, to reach a value beyond 10 for the left-most point. In this region, $\mathrm{MC}$ simulation are impeded by enhanced equilibration time (which explains why it is void of MC results).

\section{CONCLUSIONS}

We have proposed a novel procedure for solving jelliumlike models, taking due account of renormalization effects.
Such approaches had been tested with some success on liposome and latex dispersions [39,40]. Particular emphasis was put on colloidal mixtures, where it was shown that the computationally most demanding part of the task boils down to a sequence of monocomponent calculations. The idea was illustrated on binary mixtures, but can be straightforwardly generalized to arbitrary polydispersities, including continuous case after suitable discretization. The method takes advantage of the mean-field nature of the theory, where all species considered obey the same Poisson equation, with different boundary conditions, in a background density that couples all constituents of the mixture.

In a second step, we have performed Monte Carlo simulations of binary mixtures at primitive model level: the solvent is viewed as a dielectric continuum, but otherwise, Coulombic interactions are treated exactly. This allows us to assess the accuracy of mean-field simplifications. In this respect, we tested the jellium predictions for the osmotic pressure and those of the Poisson-Boltzmann cell, against Monte Carlo. It was known that in the monocomponent case, both mean-field approaches yield very close results, that fare very favorably against MC, provided, of course, one remains in the regime of relatively weak couplings where Poisson-Boltzmann theory may hold. We have shown here that despite the different nature of the jellium and Poisson-Boltzmann cell approximations, both approaches continue to give similar results, close to $\mathrm{MC}$, in the case of binary mixtures of spherical colloids.

Finally, while the method was illustrated on the simplest implementation of the jellium view, refinements and generalizations along the lines proposed in Refs. [41-43] can also be addressed. It is also of interest to extend our approach to colloidal objects of nonspherical shapes.
[1] L. Belloni, J. Phys. Condens. Matt. 12, R549 (2000).

[2] J.-P. Hansen and H. Löwen, Annu. Rev. Phys. Chem. 51, 209 (2000).

[3] Y. Levin, Rep. Prog. Phys. 65, 1577 (2002).

[4] R. Messina, J. Phys.: Condens. Matter 21, 113102 (2009).

[5] M. Fushiki, J. Chem. Phys. 97, 6700 (1992).

[6] J. Dobnikar, Y. Chen, R. Rzehak, and H. H. von Grünberg, J. Chem. Phys. 119, 4971 (2003).

[7] R. A. Marcus, J. Chem. Phys. 23, 1057 (1955).

[8] E. Trizac and Y. Levin, Phys. Rev. E 69, 031403 (2004).

[9] B. Beresford-Smith, D. Y. Chan, and D. J. Mitchell, J. Colloid Interface Sci. 105, 216 (1984).

[10] S. Pianegonda, E. Trizac, and Y. Levin, J. Chem. Phys. 126, 014702 (2007).

[11] J. M. Falcón-González and R. Castañeda-Priego, J. Chem. Phys. 133, 216101 (2010).

[12] J. M. Falcón-González and R. Castañeda-Priego, Phys. Rev. E 83, 041401 (2011).

[13] Y. Levin, E. Trizac, and L. Bocquet, J. Phys.: Condens. Matter 15, S3523 (2003).

[14] J. Dobnikar, R. Castaneda-Priego, H. H. von Grünberg, and E. Trizac, New J. Phys. 8, 277 (2006).

[15] A. R. Denton, J. Phys. Condens. Matter 20, 494230 (2008).

[16] A. R. Denton, J. Phys. Condens. Matt. 22, 364108 (2010).

[17] A. Torres, G. Téllez, and R. van Roij, J. Chem. Phys. 128, 154906 (2008).
[18] R. Krause, B. d'Aguanno, J. M. Mendez-Alcaraz, G. Nägele, and R. Klein, J. Phys. Condens. Matter 3, 4459 (1991).

[19] A.-P. Hynninen and M. Dijkstra, Phys. Rev. Lett. 94, 138303 (2005).

[20] A.-P. Hynninen, C. G. Christova, R. van Roij, A. van Blaaderen, and M. Dijkstra, Phys. Rev. Lett 96, 138308 (2006).

[21] K. Yoshizawa, N. Wakabayashi, M. Yonese, J. Yamanaka, and C. P. Royall, Soft Matter 8, 11732 (2012).

[22] S. Alexander, P. M. Chaikin, P. Grant, G. J. Morales, and P. Pincus, J. Chem. Phys. 80, 5776 (1984).

[23] E. Trizac, L. Bocquet, and M. Aubouy, Phys. Rev. Lett. 89, 248301 (2002)

[24] E. Trizac, L. Bocquet, M. Aubouy, and H. H. von Grünberg, Langmuir 19, 4027 (2003).

[25] G. Téllez and E. Trizac, Phys. Rev. E 68, 061401 (2003).

[26] M. Heinen, T. Palberg, and H. Löwen, J. Chem. Phys. 140, 124904 (2014).

[27] To this end, the system is enclosed in a spherical large enough cell of radius $\widetilde{R}$, such that $\tilde{\kappa} \widetilde{R} \gg 1$. At that point $\tilde{r}=\widetilde{R}, d \phi / d \tilde{r}$ is taken to vanish (electroneutrality), and the potential $\phi(\widetilde{R})$ is varied, in order to match a prescribed gradient at $\tilde{r}=1$, given by the bare charge (denoted $Y$ here). Under the proviso that the cell is large enough, the results found do not depend on $\widetilde{R}$, which is only used for numerical purposes. 
[28] Yet, with an asymmetric salt where co-ions have a larger valency than counter-ions, the effective charge is not always smaller than the bare one; see, e.g., Ref. [44].

[29] For instance, the parameter $\phi(\widetilde{R})$ mentioned in [27] needs to be varied.

[30] The bottom curve with $\kappa_{\text {res }} a_{1}=0.5$ exhibits a saturation value below 8 for $\widetilde{Z}_{\text {eff }}^{1}$, which should also set the upper possible limit for $\widetilde{Z}_{\text {eff }}^{2}$, since the two species are of the same size in that example. However, the a priori chosen value was $\widetilde{Z}_{\text {eff }}^{2}=9$.

[31] D. Frenkel and B. Smit, Understanding Molecular Simulation: From Algorithms to Applications (Academic Press, San Diego, 2001).

[32] C. Álvarez, Ph.D. thesis, Université Paris-Sud, no 9848 (2010).

[33] P. P. Ewald, Ann. Phys. 369, 253 (1921).

[34] S. W. de Leeuw, J. W. Perram, and E. R. Smith, Proc. R. Soc. London A 373, 27 (1980).

[35] M. P. Allen and D. J. Tildesley. Computer Simulation of Liquids (Clarendon Press, Oxford, 2001).
[36] J. W. Perram, M. S. Wertheim, J. L. Lebowitz, and G. O. Williams, Chem. Phys. Lett. 105, 277 (1984).

[37] L. Šamaj and E. Trizac, Phys. Rev. Lett. 106, 078301 (2011).

[38] Yet, it should be noted that $\Xi_{i} \propto \widetilde{Z}_{i} \ell_{B} / a_{i}$. Thus, with a small enough value of $\ell_{B} / a_{i}$, one can have both a large value of $\widetilde{Z}_{i}$ (and thus important nonlinear effects), with a small plasma parameter $\Xi_{i}$ (validating the mean-field description).

[39] C. Haro-Pérez, M. Quesada-Pérez, J. Callejas-Fernández, R. Sabate, J. Estelrich, and R. Hidalgo-Álvarez, Colloids Surf. A 270, 352 (2005).

[40] C. Haro-Pérez, M. Quesada-Pérez, J. Callejas-Fernández, P. Schurtenberger, and R. Hidalgo-Álvarez, J. Phys.: Condens. Matter 18, L363 (2006).

[41] R. Castañeda-Priego, L. F. Rojas-Ochoa, V. Lobaskin, and J. C. Mixteco-Sanchez, Phys. Rev. E 74, 051408 (2006).

[42] T. E. Colla, Y. Levin, and E. Trizac, J. Chem. Phys. 131, 074115 (2009).

[43] T. E. Colla and Y. Levin, J. Chem. Phys. 133, 234105 (2010).

[44] G. Téllez and E. Trizac, Phys. Rev. E 70, 011404 (2004). 